\title{
Patients with cardiac disease: considerations for the dental
} practitioner

\author{
N. I. Jowett,' and L. B. Cabot, ${ }^{2}$
}

The provision of dental treatment under both local anaesthesia and sedation has an excellent safety record, although medical problems may occur. The high prevalence of cardiac disease in the population, particularly ischaemic heart disease, makes it the most common medical problem encountered in dental practice. Additionally, the increasing survival of children with congenital heart disease makes them a significant proportion of those attending for dental treatment. While most dental practitioners feel confident in performing cardio-pulmonary resuscitation, treating patients with co-existent cardio-vascular disease often causes concern over potential problems during treatment. This article aims to allay many of these fears by describing the commoner cardiac conditions and how they may affect dental treatment. It outlines prophylactic and remediable measures that may be taken to enable safe delivery of dental care.

$\mathrm{T}_{\mathrm{i}}$ The high prevalence of cardiac disorders in the population, particularly ischaemic heart disease, means that the general dental practitioner will frequently encounter patients with these conditions. Chapman reported that angina was one of the more common medical emergencies encountered in the dental chair, ${ }^{1}$ the others being anaphylaxis, hypoglycaemia and seizures. Peacock and Carson found that over half the patients attending a periodontal clinic had a significant medical history, with drug allergies and cardiovascular disorders being predominant. $^{2}$ Co-existent cardiovascular disease is the most frequently cited medical condition for patient referral from general dental practitioners to hospital departments of oral and maxillo-facial surgery, which reflects widespread concern over potential problems during treatment. ${ }^{3}$ The major

${ }^{1}$ Consultant, Department of Cardiovascular

Medicine, Withybush General Hospital,

Pembrokeshire SA61 2PZ; ${ }^{*}$ Senior Lecturer,

Department of Prosthetic Dentistry, GKT Dental

Institute, Guy's Tower, London Bridge SE1 9RT

${ }^{*}$ Correspondence to: Lyndon Cabot

REFEREED PAPER

Received 19.05.99; Accepted 2.03.00

(C) British Dental Journal 2000; 189: 297-302 concerns identified are the presence of heart murmurs, the need for antibiotic therapy, hypertension and the safety of administering vaso-constrictor drugs. ${ }^{4}$ Many of these patients are subsequently found not to be at significant risk, and could easily be managed in general dental practice suggesting education is an important requirement. ${ }^{1}$ Additionally, the increasing survival of children with congenital heart disease means that a significant number of affected patients will be seen in practice.

Carrying out dental procedures on patients with cardiovascular disease can lead to difficulties. The potential problems that a dental practitioner is likely to encounter will be considered under the following headings:

- Ischaemic heart disease

- Hypertension

- Dysrhythmias and pacemakers

- Valvular heart disease

- Anticoagulants

- Congenital heart disease

- Cardioactive drugs and vasoconstrictor therapy

Because cardiac disorders are common, evaluation of the medical history is an essential initial step of any treatment plan. If the dentist has any concerns about management, he or she should not hesitate to contact the patient's general medical practitioner or physician. Good communication forms the basis of safe care.

\section{Ischaemic heart disease}

Ischaemic heart disease is common in the general population. It is therefore common in dental practice. Most stable patients will either have heart failure or angina.

Heart failure describes the inability of the heart to provide a cardiac output sufficient for metabolic needs. It is common, particularly in the elderly. It associates with a significant morbidity and mortality; in the Framingham study, the overall mortality at 2 years was $25 \%$. The overall prevalence in the general population is $1-2 \%$, and the underlying cause is usually coronary artery disease or hypertension. Symptoms are many, but the dominant features are shortness of breath, lethargy and ankle oedema. Modern drug therapy has greatly improved the outlook for affected patients, although this often involves complex drug regimes, including anticoagulants. The practitioner should therefore be particularly vigilant in identifying the medication that a patient is taking for their heart failure.

Angina affects around $1 \%$ of the population, the prevalence increasing with age. It is usually caused by coronary artery disease, and anginal pain is precipitated when there is an inadequate supply of oxygen to the myocardium. The pain is usually felt retrosternally, radiating to the neck or arms, although it is important to recognise that the perception of pain differs from one patient to the next, as does their ability to describe it. Furthermore, a patient may experience different types of chest pain, which may or may not be cardiac in origin. It should not be forgotten that anginal pain is often felt in the mandible, with secondary radiation to the neck and throat. The patient may initially suspect the pain to be 
of dental origin, presenting to a dental rather than medical practitioner.

Typically, anginal pain is precipitated by factors increasing myocardial oxygen demand. Usually this is effort, and thus the pain follows the onset of exercise, resolving within a few minutes of rest. However, the dental environment increases the likelihood of an anginal attack because of associated fear, anxiety or pain. Anxiety levels vary considerably from individual to individual, as does the response.

At-risk patients should be encouraged to bring their usual anti-anginal therapy with them. Oral nitrates are the standard emergency remedy, and should be taken either as a sub-lingual glyceryl trinitrate (GTN) tablet, a modified slow-release GTN tablet placed in the buccal sulcus (Buccal Suscard), or as a sub-lingual GTN spray. If the patient experiences an anginal attack in the chair, the treatment should stop, and the patient should be allowed to relieve the attack themselves in their usual way. Adjunctive oxygen may be used, but this is not usually required. After the attack has passed, it will normally be safe to continue dental treatment, if the patient so wishes.

Anginal pain is transient. Prolonged chest pain may suggest myocardial infarction. The pain experienced during myocardial infarction is usually severe - and it is not relieved by GTN. Co-existent nausea and sweating are common. This is a medical emergency, and while the most important intervention is to get the patient to hospital as quickly as possible, there are simple First Aid measures which may benefit the patient. It must be remembered that patients often carry drugs that are useful in an emergency. The first line drug of choice for acute myocardial infarction is Aspirin and one $300 \mathrm{mg}$ tablet should be given for the patient to chew. Chewing the Aspirin is important - it reaches the circulation quickly. GTN (either as a spray or sub-lingual tablet) should also be given as this relieves any associated spasm within the coronary vessels. The patient may well have this with them. Oxygen is very helpful, and if possible the practitioner should obtain venous access with an intravenous cannula.

\section{In brief}

For angina:

Signs and symptoms:

- Patients will recognise this for what it is

- Pain is transient, usually retro-sternal

- Pain may radiate to neck and arms Treatment:

- Allow patients to treat the attack themselves

- Normally safe to continue treatment if patient wishes

For myocardial infarction (MI): Signs and symptoms:

- Prolonged severe retro-sternal chest pain suggests $\mathrm{MI}$

- Patient may be cold, clammy, nauseous and frightened

- Pain is not relieved by GTN Treatment:

- Summon ambulance

- Give Aspirin to chew, plus GTN

- Oxygen is helpful

- Gain venous access if possible

There are three reasons for this. Firstly, cardiovascular collapse may occur at any time, and finding a vein then will be very difficult. Secondly, it provides a speedy and more reliable route for drug delivery, since patients having a heart attack frequently vomit. Thirdly, intravenous thrombolytic agents will usually be administered immediately on arrival in hospital. Intra-muscular injections produce trauma and the subsequent thrombolysis may cause large intra-muscular bleeds. Early relief of pain is also very important. Nitrous oxide/oxygen mixture, if available, may be helpful but intra-venous diamorphine and an antiemetic are optimal, and will be given on arrival in hospital if not already given by the paramedics.

Minor dental interventions seem to be well tolerated by patients with recent uncomplicated myocardial infarction, ${ }^{5}$ but more complex treatments warrant special consideration, particularly if heart failure or dysrhythmias complicated the infarct. ${ }^{6}$ Hospital consultation may then be appropriate. If general anaesthesia is being considered, there are certain clinical features which make it a significant risk. These include myocardial infarction within the previous 6 months, signs of heart failure and a history of rhythm disturbances.

\section{Patients with hypertension}

Blood pressure is variable and there is a circadian rhythm. It is lowest during the night, and highest first thing in the morning. It rises with age and with anxiety. A blood pressure of under $140 / 90-\mathrm{mm} \mathrm{Hg}$ is considered normal. Patients with blood pressures consistently above 160/90-mm $\mathrm{Hg}$ are defined as hypertensive and should receive treatment, since they are at increased risk of stroke, heart failure, myocardial infarction and renal failure. Over $95 \%$ of hypertensive patients do not have an obvious underlying cause, and are said to have essential hypertension. A few patients may have identifiable causes such as renal or endocrinological disease.

The proposed threshold for pharmacological intervention in hypertension has gradually been reduced in the light of the findings of large randomised trials. In the past, entry to such trials was based upon the diastolic blood pressure alone, but both systolic and diastolic blood pressures have been found to be of equal importance. The optimal blood pressure in a treated patient is now a systolic blood pressure $<140-\mathrm{mm} \mathrm{Hg}$ and a diastolic blood pressure $<80-\mathrm{mm} \mathrm{Hg}{ }^{7}$ As well as drug therapy, treatment usually includes advice on lifestyle changes, including cessation of smoking, weight control, and a reduction in salt, caffeine and alcohol intake. Many different drugs are used in the treatment of hypertension. Those most commonly prescribed are diuretics, beta-blockers, calcium antagonists, alpha-blockers, angiotensin converting enzyme (ACE) inhibitors and angiotensin II blockers. The efficacy of treatment depends upon many features including age and ethnic group. Drug therapy is tailored to the individual to maximise compliance, which is probably the most important factor in longterm blood pressure control.

Many patients with hypertension remain 
undiagnosed, and nearly $50 \%$ of patients on treatment are not controlled. ${ }^{8}$ A case could therefore be made for measuring blood pressure in all patients attending for dental treatment (especially those already known to be hypertensive). However, the stress of a dental visit may artificially raise the blood pressure. An increase in mean heart rate and blood pressure can be induced both by anticipation and actual dental treatment. Similar significant changes are also seen before a local anaesthetic is administered, during restorative treatment, extractions and when adrenaline-impregnated retraction cords are used. ${ }^{9}$ Although these effects are usually within normal physiological variation, they may be exaggerated in any patient with cardiovascular disease, and particularly those with hypertension. Reducing these hypertensive episodes during dental therapy may be influenced by an elimination of pain and minimising anxiety. Conscious sedation and/or hypnosis may be useful in some patients. ${ }^{10}$

\section{Patients with dysrhythmias}

A dysrhythmia describes any abnormality in the rate, regularity or site of origin of the cardiac impulse, or where there is a disturbance in the conduction of that impulse such that the normal sequence of atrial and ventricular activation is altered. Hence, dysrhythmias result from abnormalities of impulse generation, impulse conduction or both. Dysrhythmias are sometimes found in those without cardiac problems, but they are more common in those with cardiovascular disease. Whatever the aetiology, any rhythm disturbance may lead to a reduction in efficiency of the cardiac pump. Obviously, the occasional premature ventricular beat will have no significant effect on the circulation, and an increase in pulse rate (tachycardia) is the normal response of the heart to increased physical work. However abnormal tachycardias are often associated with a diminished cardiac output and sometimes with cardiovascular embarrassment. Symptoms provoked by tachycardia are determined less by the origin of the dysrhythmia and more by the heart rate and presence of underlying cardiac disease. Many episodes are asymptomatic, and extra beats are very common in normal people. However, in those with heart disease, provoked symptoms may include angina, dyspnea, palpitations or syncope.

Cardiac dysrhythmias are well recognised during oral surgery. An increase in both supra-ventricular and ventricular ectopic beats is seen in many patients with cardiovascular disease during extractions or preprosthetic surgery under local anaesthesia. ${ }^{11}$ The duration of rhythm disturbance is usually short, cardiac output is maintained, and it is normally of little consequence. It should be noted that patients treated with digoxin for atrial fibrillation or congestive cardiac failure are more prone to rhythm complications during dental extractions than other cardiac patients, ${ }^{12}$ and these patients should be considered for electrocardiographic monitoring. ${ }^{13}$

With the decline in the use of halothane the incidence of dysrhythmias under general anaesthesia has reduced dramatically. However, rhythm disturbances are not uncommon because of the mechanical stimulation of the tissues in association with the lighter plane of anaesthesia usually employed. Although these are usually transient, they may be of importance in patients with cardiac disease. The Poswillo Report recommends that all patients having general anaesthesia for dental procedures should be monitored by pulse oximetry, ECG and non-invasive blood pressure monitors. ${ }^{14}$ The combination of insufficient analgesia, adrenaline and cardiovascular disease may cause cardiac dysrhythmias, and some advise this level of monitoring to give early warning of potentially deleterious cardiovascular effects, even if treatment is scheduled under local anaesthesia. ${ }^{15}$

\section{Patients with cardiac pacemakers}

Modern cardiac pacemakers are a complex and heterogeneous group of devices, but they are usually tolerant of most external insults. If the units are functioning normally pre-operatively, they are likely to continue to do so during dental treatment. Diathermy is the main concern as it can interfere with pacemaker function. If needed, bipolar diathermy is preferred because the patient's body does not form part of the electrical cir- cuit. Other electric and electro-mechanical signals can interfere with pacemaker function, such as electronic apex locators, ultrasonic scalers, and even ultrasonic cleaning baths. However, electric pulp testers and dental hand pieces appear to be safe. ${ }^{16}$

'Rate responsive' pacemakers speed up or slow down the pulse rate in response to certain physiological stimuli, such as changes in respiratory activity or movement. These normally signal an increased heart rate during exercise, but the vibration sensors may cause inappropriate inhibition or triggering of the pacemaker during surgical procedures if vibration is induced, or even with shivering when a patient is recovering from anaesthesia. Providing this is understood, it should be possible to minimise any untoward stimuli, although the pacemaker can be reprogrammed if necessary. Patients considered to be at risk from external interference can have a magnet placed over the pacemaker to switch the pacemaker to fixed-rate mode making it immune to external signals. Overall, the risks of pacemaker malfunction are very small.

\section{Patients with valvular heart disease}

Any heart valve lesion can impair the circulation, ultimately resulting in heart failure. Such lesions are either obstructive (stenotic), or incompetent (regurgitant), and most abnormalities affect the aortic and mitral valves. Right sided valvular disease is usually secondary to pulmonary disease, although pulmonary stenosis is a relatively common congenital problem. Despite the decline in rheumatic fever, valvular heart disease remains a common and important pathology.

Many degenerating valves require operative therapy, which may involve valve reconstruction or valve replacement, using tissue or mechanical prostheses. Around 5,000 cases of valve surgery are undertaken in the UK annually, usually for calcific aortic stenosis or mitral valve degeneration. World-wide, chronic rheumatic fever is the usual underlying disease.

Biological valves are either homografts (from cadavers) or heterografts (from cattle or pigs), and are used mainly in the aortic position. Valves of animal origin are usually 
sewn onto a support frame, and referred to as bio-prosthetic valves. Mechanical valves may be used in both the aortic and mitral position and usually work either by tilting discs or consist of a cage and ball mechanism. Mechanical prostheses are very durable, but they require life long anti-coagulation to reduce the risk of thrombo-embolism or of thrombosis of the valve itself. The annual risk of thrombo-embolism in patients with mechanical valves and non-anticoagulated bio-prosthetic valves is about $2 \%$. Mitral prostheses carry twice this risk. Homografts, however do not appear to be associated with thrombo-embolic events. The degree of anticoagulation needed to protect against thrombo-embolism varies with the type of prosthesis. For mechanical valves in the aortic position, the INR (International Normalised Ratio) target is usually 2.5. In the mitral position, the target is somewhat higher at 3.5, since flow through this valve is slower. This is particularly significant when considering dental treatment (see next section).

Oral anticoagulants are not required for bio-prosthetic valves in the aortic position if the patient is in sinus rhythm, although most cardiac centres will normally anticoagulate a patient for 3-6 months following surgery. Bio-prosthetic valves in the mitral position sometimes require longterm anticoagulation. Bio-prosthetic valves have limited durability (7-10 years), and repeat surgery is often needed.

There are two main concerns during dental treatment of patients with valvular disease:

- The risk of infective endocarditis

- The risk of bleeding in anticoagulated patients.

\section{The risk of endocarditis}

Endocarditis is more likely to occur in patients who have previously had endocarditis and those with certain cardiac lesions (Table 1). The risk of endocarditis for patients with prosthetic heart valves is about $2 \%$ per annum for aortic valve replacement and $0.5 \%$ per annum for mitral valve replacement. While the risk of a normally functioning prosthesis being infected after a dental procedure is probably no higher than the risk in patients with damaged native valves, it

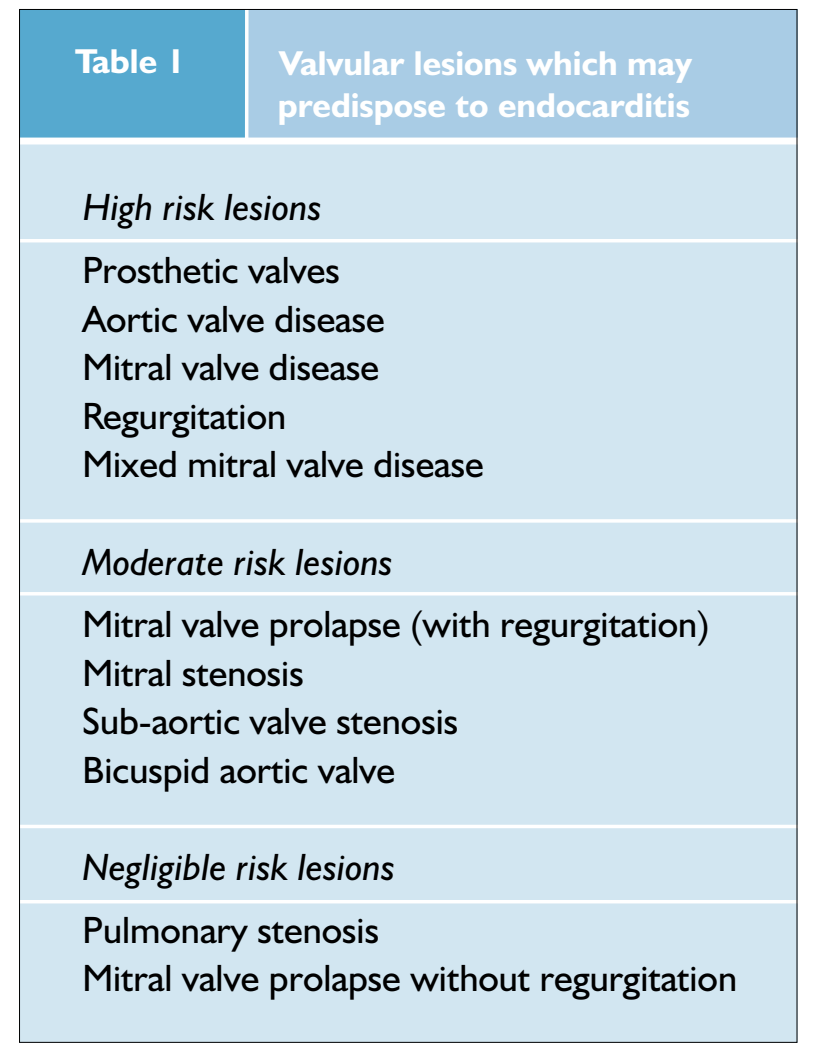

should be noted that the morbidity and mortality is much higher should the prosthesis become infected (around 50\%).

\section{The risk of bleeding}

Patients with native valve disease can often stop or reduce their anti-coagulants, but those with prosthetic valves should not discontinue their anticoagulants without taking cardiological advice. Mechanical mitral valves are prone to thrombosis, which cause emboli if adequate anti-coagulation is not maintained, although short term modification may be possible. ${ }^{17}$

\section{Anti-coagulants}

Apart from those with prosthetic heart valves, anti-coagulants are frequently prescribed for many other categories of cardiovascular disease, such as heart failure, atrial fibrillation and deep vein thrombosis. The decision to anti-coagulate patients is usually based upon the estimated risk of thrombo-embolism against any side effects of treatment, which essentially means unwanted bleeding. Factors increasing this risk include concomitant disease (particularly renal and liver disease), a history of gastro-intestinal bleeding, poor mobility with falls, misuse of alcohol and poor compliance with medication.

Different INR targets are set for different conditions, but for practical purposes, most patients aim for a target value of 2.5. Most dental procedures can be safely performed without having to withdraw anticoagulant therapy, provided the INR is 2.5 or below. ${ }^{18}$ However, as noted in the previous section, the target INR for some patients can be somewhat higher. The risk of lowering a patient's INR below the therapeutic range needs to be weighed against the risks of thrombosis. Close liaison with the patient's physician is therefore very important. In any event, it is of paramount importance to obtain an assessment of the current level of anti-coagulation with an INR test as close to the planned procedure as possible. If short term anticoagulant therapy has been prescribed, for example 
in the treatment of deep vein thrombosis or pulmonary embolus, elective dental treatment is best delayed until the end of therapy. Apart from Warfarin, aspirin and other anti-platelet agents (eg Persantin, clopidogrel) are commonly prescribed for many cardiac disorders, and may cause prolonged bleeding and this should be taken into account before any surgical procedures are undertaken.

Blood loss during and after oral surgery on anticoagulated patients may be significant, but most bleeding incidents are not serious and can be controlled by local measures. ${ }^{19}$ An anti-fibrinolytic mouthwash containing tranexamic acid (4.5\%) can effectively suppress post-operative bleeding without needing to modify the dose of warfarin, ${ }^{20}$ and gelatin sponges, oxidised cellulose and micro crystalline collagen are other useful haemostatic agents.

The practitioner should be aware that many drugs can interact with anticoagulants, causing derangement of anti-coagulation. For example, potentially serious interactions have been described with miconazole oral gel. ${ }^{21}$ However, unless any modification of drug therapy is intended to last for longer than 5 days, either no change or minor anticoagulant dose reductions are required. If in doubt, it is wise to consult with a pharmacist or the patient's physician before advising changes in drug therapy. Further details may be found the appendix in the British Dental Formulary.

Some dentists give intra-muscular injections of non-steroidal anti-inflammatory agents at the end of surgery (eg ketorolac) for postoperative analgesia. They should be aware that this might be complicated by an intra-muscular hamatum in patients who are anti-coagulated.

\section{Patients with congenital heart disease}

Congenital heart disease is one of the most common developmental anomalies. While many such conditions are apparent in the neonate, a significant proportion do not come to light until the child is older, or even later on in adult life. Furthermore, many children who historically would have died either because of an undiagnosed anomaly or because of technical inability to deal with the problem now survive into adulthood. The dental practitioner may now encounter children or adults with such lesions as congenital aortic or pulmonary stenosis, atrial septal defects (ASD) or ventriculo-septal defects (VSD). Up to half of all VSDs close spontaneously, and cause little or no cardiovascular symptoms. Surgery is usual for ASDs, although many are not diagnosed until middle age. A patent foramen ovale may be found in up to a quarter of all children. They do not associate with much inter-atrial shunting, do not really require endocarditis prophylaxis and rarely need surgical repair.

Children with congenital heart disease require special care dentistry because of their susceptibility to infective endocarditis. Unfortunately, many of these children are found to have poor oral health with high levels of dental caries and untreated gingivitis. ${ }^{22}$ They also seem to receive less than optimal professional and home dental care. $^{23,24}$ While cardiologists usually advise the parents of children with heart disease to attend their dentist regularly, such advice does not seem to be followed. Visual prompts such as the British Heart Foundation 'heart card', which details the patient's cardiac defect and appropriate antibiotic prophylaxis if required, do not seem to help. The cards are often lost, or the advice contained therein is simply not followed. In a study of 60 children with severe congenital heart disease, $18 \%$ had not even visited a dentist. ${ }^{25}$

With advances in cardiac care and cardiac surgery, many children are surviving to lead normal adult lives. Most cardiac units now run GUCH (grown up congenital heart disease) clinics to monitor and advise patients and their families. Unfortunately, growing up does not seem to have an impact on their level of understanding in terms of either their diagnosis or risk of endocarditis. ${ }^{26}$ Many patients believe that surgery has negated the risk of endocarditis, which may or may not be the case. Certainly, surgically corrected septal defects are at a negligible risk of endocarditis, but if there are post-surgical shunts or conduits, the risk remains. With the increase in surgery for congenital cardiac disease, and utilisation of intra-cardiac prosthetic material, a new 'at risk' sub-group has emerged.

\section{Cardio-active drugs and the safety of vasoconstrictor therapy}

Patients with cardiovascular disease often take a lot of medication. Following an uncomplicated myocardial infarction, it is not unusual for a patient to be discharged from hospital taking aspirin, a beta-adrenergic blocker, as well as cholesterol lowering medication. Patients with heart failure may be on all of these, plus an ACE inhibitor, digoxin and warfarin. The potential risk from drug interaction during dental treatment must be considered. For example, if adrenaline-containing local anaesthetics are administered to patients taking beta-blockers, a drug interaction could induce a steep rise in blood pressure, which could trigger cardiovascular complications. ${ }^{27}$ Cases of severe headache have been documented following administration of noradrenalinecontaining anaesthetic thought to be caused by a transient acute hypertensive episode. During these steep rises in blood pressure, angina or disturbances of cardiac rhythm may also be precipitated, and the use of a vasoconstrictor should be considered very carefully in patients with heart disease, particularly angina, recent myocardial infarction or cardiac surgery, untreated or uncontrolled hypertension and heart failure. ${ }^{28}$ Accidental intra-vascular injection or rapid systemic absorption could also directly induce circulatory problems in these patients.

Oral reactions to prescribed drugs, including altered taste, impaired salivary function and gingival hyperplasia may be seen in cardiac patients. ${ }^{29}$ While abnormal growth of the periodontal tissue is mainly associated with plaque related inflammation, drugs such as nifedipine and amlodipine, have been implicated in causing gingival overgrowth, which may be brought to dental attention because of pain, bleeding or appearance. ${ }^{30}$ The anti-dysrhythmic agents disopyramide and propafenone and the anti-hypertensive drugs indoramin and methyldopa may cause a dry mouth. ACE inhibitors and amiodarone sometimes give rise to altered taste, 
typically described as 'metallic'. If an offending drug can be identified, it may be possible (in consultation with the prescriber) to discontinue it or give an alternative.

\section{Summary}

The provision of dental treatment under local anaesthesia with or without sedation has an excellent safety record, although medical problems may occur. This may become increasingly common as our patient population ages, cardiac disease being the most common cause of morbidity and mortality in the elderly.

Being forewarned, taking account of the previous medical history and medical evaluation are essential considerations for safe delivery of care. It is useful to establish current medication and allergies and to note any potential drug interactions and side effects. Some patients may require prior medical assessment or planned treatment following discussions with their physician. Many patients fast before dental treatment under the misconception that this is necessary.

If anti-hypertensive or anti-anginal therapy is omitted, it may precipitate cardiovascular complications during dental treatment. It is therefore important to ensure the patient has not abruptly discontinued their medication. Routine blood pressure measurement in particular will help screen for undiagnosed hypertension.

Pre-medication should be considered to alleviate anxiety and effective analgesia is important to reduce stress. Conscious sedation may be considered, particularly for those with coronary artery disease. Special consideration should be given as to whether adrenaline-containing local anaesthetics are really necessary in view of the potential problems, and impregnated gingival displacement cords should be avoided in those with hypertension and ischaemic heart disease.

Ensuring a fresh supply of GTN is desirable for patients with angina, and this can also be used if hypertension is a problem during dental treatment. It is helpful to ask patients to bring their own usual medication.

Morning appointments are preferable; there is less time for the patient to become anxious, and more time to take effective mea- sures if peri-treatment problems occur. Every effort should be made to keep procedure time down to a minimum, and treatment should be terminated early if the patient becomes overly anxious. If angina or other significant symptoms develop, all treatment should stop, and the practitioner should be prepared to initiate emergency care.

1 Chapman P J. Medical emergencies in dental practice and choice of emergency drugs and equipment: a survey of Australian dentists. Aust Dent J 1997; 42:103-108.

2 Peacock M E, Carson R E. Frequency of selfreported medical conditions in periodontal patients. J Periodontol 1995; 66: 1004-1007.

3 Absi E G, Satterthwaite J, Shepherd J P, Thomas $\mathrm{D} W$. The appropriateness of referral of medically compromised dental patients to hospital. Br J Oral Maxillofac Surg 1997; 35 : 133-136.

4 Jainkittivong A, Yeh C K, Guest G F, Cottone $\mathrm{J}$ A. Evaluation of medical consultations in a predoctoral dental clinic. Oral Surg Oral Med Oral Pathol Oral Radiol Endod 1995; 80: 409. 413.

5 Cintron G, Medina R, Reyes A A, Lyman G. Cardiovascular effects and safety of dental anaesthesia and dental interventions in patients with recent uncomplicated myocardial infarction. Arch Intern Med 1986; 146: 2203 2204.

6 McCarthy F M. Safe treatment of the post heart attack patient. Compend Contin Educ Dent 1989; 10: 598-604.

7 Hansson L, Zanchetti A, Carruthers S G. Effects of intensive blood pressure lowering and low dose aspirin in patients with hypertension: principle results of the hypertension optimisation treatment (HOT) randomised trial. Lancet 1998; 351: 1755-1762.

8 Colhoun H M, Dong W, Poulter N R. Blood pressure screening, management and control in England 1994. J Hypertension 1998; 16: 747753.

9 Brand H S, Abraham-Inpijn L. Cardiovascular responses induced by dental treatment. Eur J Oral Sc 1996; 104: 245-252.

10 Lu D P, Lu G P. Hypnosis and pharmacological sedation for medically compromised patients. Compend Contin Educ Dent 1996; 17: 32-40.

11 Campbell R L, Langston W G, Ross G A. A comparison of cardiac rate-pressure product and pressure-rate quotient with Holter monitoring in patients with hypertension and cardiovascular disease: a follow-up report. Ora Surg Oral Med Oral Pathol Oral Radiol Endod 1997; 84: 125-128.

12 Blinder D, Shemesh J, Taicher S. Electrocardiographic changes in cardiac patients undergoing dental extractions under local anaesthesia. J Oral Maxillofac Surg 1996; 54: 162-165.

13 Jowett N I. Electrocardiographic monitoring. In: Cardiovascular Monitoring 1997. London: Whurr Publishers, pp: 35-61.
14 General anaesthesia, sedation and resuscitation in dentistry. Report of an expert working party. London: Department of Health, 1990.

15 Umino M, Ohwatari T, Shimoyama K, Nagao M. Unexpected atrial fibrillation during tooth extraction in a sedated elderly patient. Anaesthesia Progress 1994; 41: 77-80.

16 Miller C S, Leonelli F M, and Latham E. Selective interference with pacemaker activity by electrical dental devices. Oral Surg Oral Med Oral Pathol Oral Radiol \& Endod 1998; 85. 33-36.

17 Tinker J H, Tarhan S. Discontinuing anticoagulant therapy in surgical patients with cardiac valve prostheses. J Am Med Ass 1978; 239: 738.

18 Purcell C A. Dental management of the anticoagulated patient. N Z Dent J 1997; 93: 87-92.

19 Weibert R T. Oral anti-coagulation therapy in patients undergoing dental surgery. Clin Pharmacy 1992; 11: 857-864.

20 Ranstrom G, Sindet-Pedersen S, Hall G, Blomback M, Alander U. Prevention of postsurgical bleeding in oral surgery using tranexamic acid without dose modification of oral anticoagulants. J Oral Maxillofac Surg 1993; 51: 1211-1216.

21 Pemberton M N, Sloan P, Ariyaratnam S, Thakker N S, Thornhill M H. Derangement of warfarin anti-coagulation by miconazole oral gel. Br Dent J 1998; 184: 68-69.

22 Franco F, Saunders C P, Roberts G J, Suwanprasit A. Dental disease, caries related microflora and salivary IgA of children with severe congenital cardiac disease: an epidemiological and oral microbial survey. Pediatr Dent 1996; 18: 228-235.

23 Hallett K B, Radford D J, Seow W K. Oral health of children with congenital cardiac diseases: a controlled study. Pediatr Dent 1992; 4: 224-230.

24 Droz D, Koch L, Lenain A, Michalski H. Bacterial endocarditis: results of a survey in a children's hospital in France. Br Dent J 1997; 183: 101-105.

25 Saunders C P, Roberts G J. Dental attitudes, knowledge and health practices of parents of children with congenital heart disease. Arch Dis Child 1997; 76: 539-540.

26 Cetta F, Warnes C A. Adults with congenital heart disease: patient knowledge of endocarditis prophylaxis. Mayo Clin Proc 1995; 70: 50-54.

27 Goulet J P, Perusse R, Turcotte J Y. Contraindications to vasoconstrictors in dentistry: Part III. Pharmacologic interactions. Oral Surg Oral Med Oral Pathol 1993; 74: 692-697.

28 Perusse R, Goulet J P, Turcotte J Y. Contraindications to vasoconstrictors in dentistry: Part 1. Cardiovascular diseases. Oral Surg Oral Med Oral Pathol 1993; 74: 679-686.

29 Wright J M. Oral manifestations of drug reactions. Dent Clin North Am 1984; 28 : 529-543.

30 Seymour R A. Calcium channel blockers and gingival overgrowth. Br Dent J 1991; 170: 376-379. 